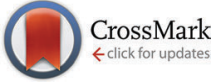

Cite this: J. Mater. Chem. C, 2017, 5,376

Received 26th October 2016, Accepted 2nd December 2016

DOI: $10.1039 / c 6 t c 04640 a$

www.rsc.org/MaterialsC

\section{Modulation doping of transition metal dichalcogenide/oxide heterostructures $\dagger$}

\begin{abstract}
Kang Xu, Yi Wang, Yuda Zhao and Yang Chai*
Control of carrier type and carrier density provides a way to tune the physical properties of twodimensional (2D) semiconductors. Modulation doping of heterostructures can effectively inject carriers into or extract carriers from the 2D semiconductors, and eliminate the adverse effect from the ionized dopants. Here we first investigate the layer-dependent negative trion $\mathrm{PL}$ of $2 \mathrm{D} \mathrm{MoS}_{2}$, and further construct heterostructures with transition metal dichalcogenides (TMDs) and transition metal oxides (TMOs). By choosing the oxide with different charge neutrality levels (CNLs), we demonstrate effective electron injection into $\mathrm{MOS}_{2}$ by $\mathrm{TiO}_{2}$ doping, and electron extraction from $\mathrm{MoS}_{2}$ by $\mathrm{MoO}_{3}$ doping. Photoluminescence (PL) spectra and electrical characterization show that thicker $\mathrm{MoS}_{2}$ flakes are more easily $n$-doped by $\mathrm{TiO}_{2}$, while thinner $\mathrm{MoS}_{2}$ flakes are more easily $\mathrm{p}$-doped by $\mathrm{MoO}_{3}$. Our experimental results are in good agreement with theoretical calculations. The modulation doping with TMO is compatible with conventional Si processing and highly air-stable. This method can also be extended for the controllable doping of other $2 \mathrm{D}$ materials.
\end{abstract}

\section{Introduction}

Two-dimensional (2D) layered transition metal dichalcogenides (TMDs) are of great research interest because of their unique electronic, optical and optoelectronic properties. ${ }^{1-5}$ The modulation of carrier density in the TMDs provides a way to change their physical properties in a controllable manner. For typical TMD materials, the pristine carrier type is predominated by their intrinsic characteristics. For example, pristine $\mathrm{MoS}_{2}$ is of the n-type while $\mathrm{WSe}_{2}$ shows p-type or ambipolar characteristics (the carrier type of $\mathrm{WSe}_{2}$ is dependent on its thickness). ${ }^{2,6-8}$ Controllable injection and extraction of electrons/holes are important for effective n- and p-doping of TMDs, which are crucial for building complementary logic circuits in the future. On the other hand, the interaction between carriers and excitons enables an additional path for the PL modulation of 2D layered materials. Controlling the carrier type and density through modulation doping provides a way to switch between exciton- and trion-dominant PL and tune the PL intensity of $2 \mathrm{D}$ materials. ${ }^{9}$

Various methods have been developed to dope 2D layered TMDs, ${ }^{10}$ including substitutional doping during growth, ${ }^{11}$ ion implantation, ${ }^{12}$ plasma treating, ${ }^{13}$ etc. Although these doping methods have been demonstrated to be effective, they inevitably result in the distortion of TMD crystal structures, introduce

Department of Applied Physics, The Hong Kong Polytechnic University, Hung Hom, Kowloon, Hong Kong, P. R. China. E-mail: ychai@polyu.edu.hk

$\dagger$ Electronic supplementary information (ESI) available. See DOI: 10.1039/ c6tc04640a ionized impurity scattering canters, and degrade the charge mobility. Modulation doping has been widely used in III-V compound heterojunction superlattices to eliminate the influence of ionized impurity scattering. ${ }^{14-16}$ In the heterojunction device, the ionized dopants and electrons are confined to two different adjacent layers. As a result, both the mobility and carrier concentration are well maintained. A variety of dopants have been studied for the surface charge transfer to TMDs, including gas molecules, ${ }^{17}$ organics, ${ }^{18}$ metal particles ${ }^{19}$ and oxides. ${ }^{2,20}$ Among these kinds of dopants, oxide is one of the promising species due to its stability in ambience and compatibility with conventional Si processing technology.

In this work, we deposit transition metal oxide (TMO) on top of $2 \mathrm{D} \mathrm{MoS}_{2}$ to form a heterojunction device. We choose $\mathrm{TiO}_{2}$ with a high charge neutrality level (CNL) and $\mathrm{MoO}_{3}$ with a low CNL for injecting and extracting electrons, respectively. Our optical and electrical characterization, together with density functional theory (DFT) calculations, shows that $\mathrm{TiO}_{2}$ and $\mathrm{MoO}_{3}$ deposition gives rise to effective n-doping and p-doping, respectively. In addition, we also reveal layer-dependent negative trion photoluminescence (PL) and doping effects in 2D layered $\mathrm{MoS}_{2}$. This approach opens up a way to control the characteristics of PL and the electrical transport of $2 \mathrm{D}$ layered materials.

\section{Results and discussion}

\section{Layer-dependent negative trion PL of 2D $\mathrm{MoS}_{2}$}

Because of the extremely strong Coulomb interaction in ultrathin 2D $\mathrm{MoS}_{2}$, optically generated excitons (i.e., electron-hole pairs) 
and trions (i.e., charged excitons) can exist stably at room temperature. ${ }^{21}$ Electrons are excited to the conducting band, leaving holes in the valence band. Electrons and holes are then bound together by Coulomb interactions to form neutrally charged excitons (electron-hole pairs). Due to the Coulomb attraction between the hole in an exciton and a third electron, the formation process of exciton to trion is energy favourable. ${ }^{22}$ Trions are formed in the presence of residual free charges. Fig. 1(a) shows the PL spectra of a $3 \mathrm{~L} \mathrm{MoS}_{2}$ as an example. Two pronounced peaks around $1.87 \mathrm{eV}$ and $2.05 \mathrm{eV}$ are clearly observed, defined as the A exciton peak and the B exciton peak, respectively. As schematically depicted in the inset, the A exciton has originated from the radiative recombination of the excited electrons in the conduction band minimum (CBM) with the holes in the valence band minimum (VBM); and the B exciton has originated from the radiative recombination of the excited electrons in the CBM with the lower branch of the split VBM. ${ }^{23}$ The A exciton of ultrathin $\mathrm{MoS}_{2}$ can be further devolved into a neutron exciton $(\mathrm{X})$ peak around $1.9 \mathrm{eV}$ and a negative trion $\left(\mathrm{X}^{-}\right)$ peak around $1.85 \mathrm{eV}^{21}$

Fig. 1(b) shows the PL spectra of $\mathrm{MoS}_{2}$ evolved with the layer number from $1 \mathrm{~L}$ to $4 \mathrm{~L}$. The PL spectra of over $60 \mathrm{MoS}_{2}$ flakes were collected and are summarized in Fig. 1(c) and (d). Fig. 1(c) shows the layer-dependent spectral weight of the $\mathrm{X}^{-}$peak in the whole A exciton peak, which rapidly decreases from $90 \%$ in the $1 \mathrm{~L} \mathrm{MoS}_{2}$ to $30 \%$ in the $3 \mathrm{~L}-5 \mathrm{~L} \mathrm{MoS}_{2}$. There are two possible reasons accounting for the layer-dependent characteristic of the spectral weight of the $\mathrm{X}^{-}$peak. First, electrons in $\mathrm{MoS}_{2}$ originated from donor states introduced by surface sulfur vacancies, ${ }^{24-27}$ which are expected to become less prominent with the increase of body thickness. Second, when the thickness of $\mathrm{MoS}_{2}$ increases, the dielectric screening of $\mathrm{MoS}_{2}$ increases, ${ }^{28,29}$ which attenuates the Coulomb interaction ${ }^{30}$ and reduces the binding energy of trions. With the decrease of binding energy,
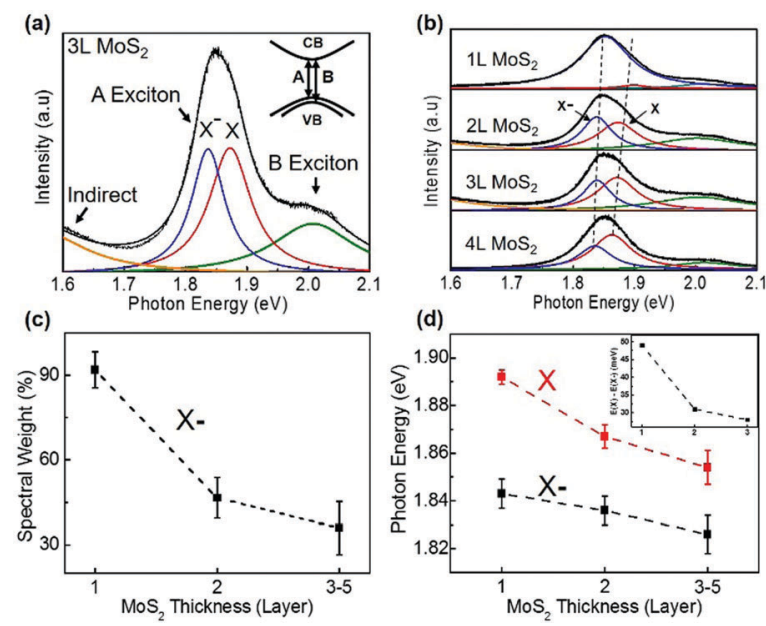

Fig. $1 \mathrm{PL}$ analysis of ultrathin $\mathrm{MoS}_{2}$ flakes. (a) PL spectrum of $3 \mathrm{LMOS}_{2}$. The inset shows the radiative recombination path of $A$ and $B$ excitons, respectively. (b) PL spectra of $1 \mathrm{~L}-4 \mathrm{~L} \mathrm{MoS}_{2}$. (c) Layer-dependent spectral weight of the $X^{-}$peak. (d) Layer-dependent photon energy of the $X$ peak and the $X^{-}$peak in the $\mathrm{MoS}_{2}$ samples from $1 \mathrm{~L}$ to $5 \mathrm{~L}$. The inset shows the layerdependent energy difference between the $X$ peak and the $X^{-}$peak. trion systems are less stable and can be easily decomposed into excitons. Fig. 1(d) shows the photon energy of $\mathrm{X}$ and $\mathrm{X}^{-}$peaks as a function of $\mathrm{MoS}_{2}$ thickness. The PL peaks exhibit a clear red-shift with an increase of $\mathrm{MoS}_{2}$ body thickness due to the decreased bandgap. In the inset of Fig. 1(d), we present the layer-dependent energy difference (binding energy of the negative trion from the exciton) between $\mathrm{X}$ and $\mathrm{X}^{-}$peaks. This binding energy decreases with the increase of $\mathrm{MoS}_{2}$ thickness.

\section{Layer-dependent n-doping of $\mathrm{MoS}_{2}$ by $\mathrm{TiO}_{2}$}

Fig. 2 shows the PL spectra of $1 \mathrm{~L}-4 \mathrm{~L} \mathrm{MoS}_{2}$ before and after $\mathrm{TiO}_{2}$ deposition. After $6 \mathrm{~nm}$ thick Atomic Layer Deposition (ALD) $\mathrm{TiO}_{2}$ doping, we observe the characteristics of layer-dependent PL. The thicker $\mathrm{MoS}_{2}$ sample has a smaller $\mathrm{X}^{-}$spectral weight before the $\mathrm{TiO}_{2}$ deposition, which is consistent with our previous discussions; while the $\mathrm{X}^{-}$spectral weight increases more after the $\mathrm{TiO}_{2}$ deposition. These changes in PL spectra are ascribed to the injection of electrons from $\mathrm{TiO}_{2}$ into $\mathrm{MoS}_{2}$, where the electron density in $\mathrm{MoS}_{2}$ is increased by $\mathrm{TiO}_{2}$ doping.

To rule out the possibility that this PL change has originated from in-plain lattice strain due to oxide deposition on the surface, we also present the corresponding Raman spectra before and after doping in Fig. S1 (ESI $\dagger$ ). As widely reported by previous research studies, the in-plane lattice strain induces the split of the $E_{2 g}^{1}$ peak and the blue-shift of both $\mathrm{E}_{2 \mathrm{~g}}^{1}$ and $\mathrm{A}_{1 \mathrm{~g}}$ peaks. ${ }^{31}$ However, from our Raman spectra, we observe no such Raman spectral change. Thus, it is suggested that the interlayer coupling between the $\mathrm{TiO}_{2}$ layer and the $\mathrm{MoS}_{2}$ surface is very weak, and this weak interaction induces negligible lattice strain to $\mathrm{MoS}_{2}$. So the PL spectral change has not originated from the lattice strain. To confirm this speculation, we further deposited $30 \mathrm{~nm}$ thick $\mathrm{TiO}_{2}$ onto $1 \mathrm{~L}-3 \mathrm{~L} \mathrm{MoS}$. The Raman and PL spectra are shown in Fig. S2 (ESI †). We still observe a more significant increase of the $\mathrm{X}^{-}$spectral weight in thicker $\mathrm{MoS}_{2}$ and a negligible shift of Raman peaks. It is noteworthy that the attenuation of Raman intensity after the doping is possibly due to the block of light of the $30 \mathrm{~nm}$ thick $\mathrm{TiO}_{2}$ layer. On the other hand, the change of the dielectric environment introduced by $\mathrm{TiO}_{2}$
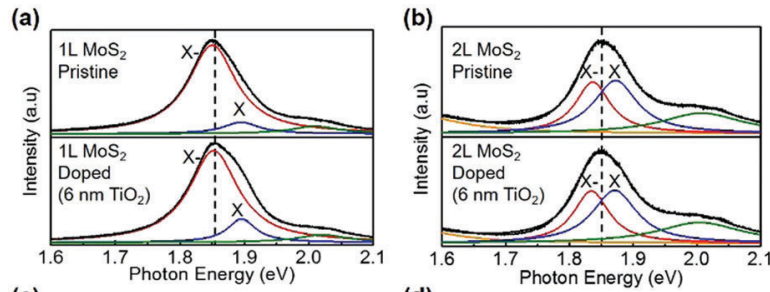

(c)

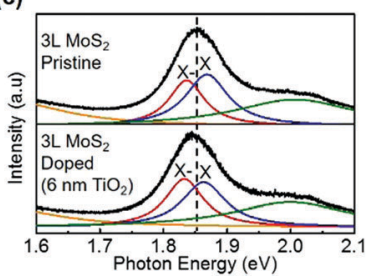

(d)

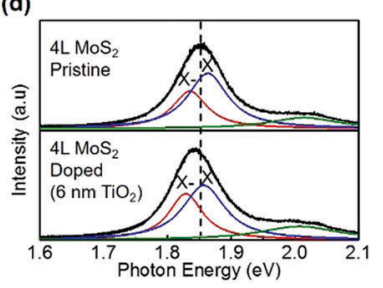

Fig. 2 PL spectra of ultrathin $\mathrm{MoS}_{2}$ flakes before and after depositing $6 \mathrm{~nm}$ thick $\mathrm{TiO}_{2}$. The thicknesses of $\mathrm{MoS}_{2}$ samples are (a) $1 \mathrm{~L}$, (b) $2 \mathrm{~L}$, (c) $3 \mathrm{~L}$ and (d) $4 \mathrm{~L}$. 
deposition plays an important role in the change of the PL spectra of $\mathrm{MoS}_{2}$. In both cases (6 nm thick and $30 \mathrm{~nm}$ thick $\mathrm{TiO}_{2}$ ), the A exciton peak is found to be red-shifted after the $\mathrm{TiO}_{2}$ deposition, which is more obvious in the case of $30 \mathrm{~nm}$ thick $\mathrm{TiO}_{2}$ deposition. This is because the bandgap of $\mathrm{MoS}_{2}$ is decreased in the environment with a higher dielectric constant (the dielectric constant of $\mathrm{TiO}_{2}$ is $\left.\sim 80\right) .{ }^{30}$ Furthermore, from the point of view of enhanced dielectric screening in $\mathrm{MoS}_{2}$ due to the deposition of the $\mathrm{TiO}_{2}$ layer, the Coulomb interaction in $\mathrm{MoS}_{2}$ is attenuated (similar to the effect of increasing $\mathrm{MoS}_{2}$ body thickness). The attenuated Coulomb interaction decreases the binding energy of the negative trion, thus decreasing the stability of the trion system and the PL intensity of the $\mathrm{X}^{-}$peak. However, in our experiments, the PL intensity of the $\mathrm{X}^{-}$peak is obviously increased after the $\mathrm{TiO}_{2}$ doping. Therefore, the increase of the $\mathrm{X}^{-}$peak PL is due to the increase of electron density in $\mathrm{MoS}_{2}$, which originated from the n-doping effect by $\mathrm{TiO}_{2}$.

To further confirm and quantify the n-doping effect, we fabricated a back-gated $\mathrm{MoS}_{2}$ field effect transistor (FET) and doped it with $6 \mathrm{~nm}$ thick $\mathrm{ALD} \mathrm{TiO}_{2}$. Fig. 3(a) depicts the crosssectional schematic of a back-gated $\mathrm{MoS}_{2}$ FET with its channel covered by TMO. Fig. 3(b) shows the transfer curve of a $5 \mathrm{~L} \mathrm{MoS}_{2}$ FET before (black line) and after (red line) the $6 \mathrm{~nm}$ thick $\mathrm{TiO}_{2}$ doping. The pristine device shows typical n-type behaviour, consistent with previous reports. ${ }^{1,32}$ After n-doping, the ON current $\left(I_{\mathrm{ON}}\right)$ is increased by a factor of 4 at $V_{\mathrm{bg}}=50 \mathrm{~V}$. Fig. 3(c) and (d) show the output curves of the same device before and after doping, respectively. Again, $I_{\mathrm{ON}}$ at $V_{\mathrm{bg}}=50 \mathrm{~V}$ is found to be amplified about 5 times. It is noteworthy that the device retains its semiconducting characteristics after the doping, indicating that the n-doping effect is non-degenerate. Furthermore, we can extract $2 \mathrm{D}$ sheet carrier density $\left(n_{2 \mathrm{D}}\right)$ and field-effect mobility $\left(\mu_{\mathrm{FE}}\right)$ according to eqn (1) and (2), where $L$ and $W$ are the channel length and the channel width, respectively, and $C_{\mathrm{ox}}$ is the capacitance of the gated oxide (i.e., $1.16 \times 10^{-8} \mathrm{~F} \mathrm{~cm}^{-2}$ (a)
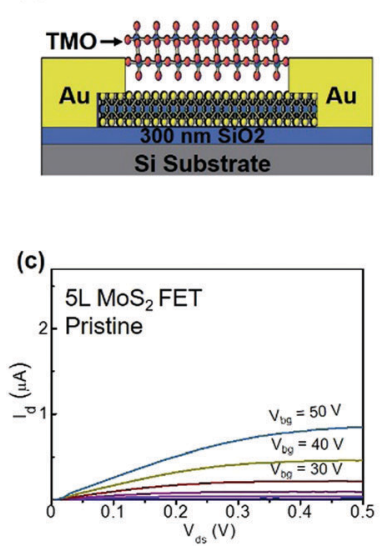

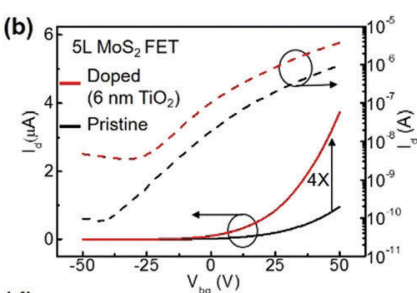

(d)

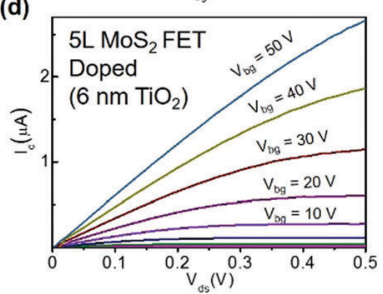

Fig. 3 Electrical characterization of a $\mathrm{MOS}_{2}$ FET for $n$-doping by $\mathrm{TiO}_{2}$ (a) Cross-sectional schematic of a TMO doped $\mathrm{MOS}_{2}$ FET. (b) Transfer curve of a $5 \mathrm{~L} \mathrm{MoS}_{2}$ FET before (black line) and after (red line) being doped by $6 \mathrm{~nm}$ thick $\mathrm{TiO}_{2}$. (c) Output curves of the FET before doping; (d) output curves of the FET after doping. for $300 \mathrm{~nm}$ thick $\mathrm{SiO}_{2}$ in this study). ${ }^{1}$ The extracted $n_{2 \mathrm{D}}$ before doping is $1.17 \times 10^{12} \mathrm{~cm}^{-2}$, which is consistent with previous reports, ${ }^{32-35}$ while the extracted $n_{2 \mathrm{D}}$ after the doping is $1.95 \times$ $10^{12} \mathrm{~cm}^{-2}$. This doping level $\left(0.78 \times 10^{12} \mathrm{~cm}^{-2}\right)$ is comparable with other doping methods (i.e., $2 \times 10^{11} \mathrm{~cm}^{-2}$ for OTS-doped $\mathrm{WSe}_{2},{ }^{36} 4.6 \times 10^{11} \mathrm{~cm}^{-2}$ for MEA-doped $\mathrm{MoS}_{2},{ }^{37}-2 \times 10^{11} \mathrm{~cm}^{-2}$ for FDT-doped $\mathrm{MoS}_{2}{ }^{34}$ and $1 \times 10^{13} \mathrm{~cm}^{-2}$ for $\mathrm{TiO}_{x}$-doped graphene). ${ }^{37}$ The $\mu_{\mathrm{FE}}$ and $n_{2 \mathrm{D}}$ are extracted at $V_{\mathrm{bg}}=30 \mathrm{~V}$. Although the doping effect is independent of the back gate voltage, sufficient gate voltage is required to turn on the channel of the device. It is noteworthy that in these devices, the charges are free carriers without the formation of excitons or trions. Because there is no external input to excite electrons to the conduction band and the lifetimes of excitons and trions are very short, in the order of picoseconds, ${ }^{38,39}$

$$
\begin{gathered}
\mu_{\mathrm{FE}}=\frac{\mathrm{d}}{\mathrm{d} V_{\mathrm{g}}} I_{\mathrm{ds}} \times \frac{L}{W \times C_{\mathrm{ox}} \times V_{\mathrm{ds}}} \\
n_{2 \mathrm{D}}=\frac{I_{\mathrm{ds}} \times L}{W \times \mu_{\mathrm{FE}} \times V_{\mathrm{ds}}}
\end{gathered}
$$

To further support our findings, DFT calculations were utilized to simulate the doping effect. The anatase phase $\mathrm{TiO}_{2}$ structure was used in this simulation since it is a stable phase at a relatively low temperature. ${ }^{20}$ Fig. 4 (a) and (b) show the atomic and orbital projected density of states of pristine $1 \mathrm{H}-\mathrm{MoS}_{2}$, $1 \mathrm{H}-\mathrm{MoS}_{2}$ in the $\mathrm{TiO}_{2}$-doped $\mathrm{MoS}_{2}$ system, anatase $\mathrm{TiO}_{2}$, and anatase $\mathrm{TiO}_{2}$ in the $\mathrm{TiO}_{2}$-doped $\mathrm{MoS}_{2}$ system. The bandgap of pristine $1 \mathrm{H}-\mathrm{MoS}_{2}$ is calculated to be $\sim 1.86 \mathrm{eV}$. And both the conduction band minimum (CBM) and the valence band maximum (VBM) of $\mathrm{MoS}_{2}$ are populated by the $4 \mathrm{~d}$ orbital of the Mo atom, which are highly hybridized with the 3p orbital of the $\mathrm{S}$ atom. ${ }^{20}$ After the $\mathrm{TiO}_{2}$ doping, the Fermi level of $1 \mathrm{~L} \mathrm{MoS}_{2}$ is up-shifted approximately $0.76 \mathrm{eV}$ towards the CBM, indicating
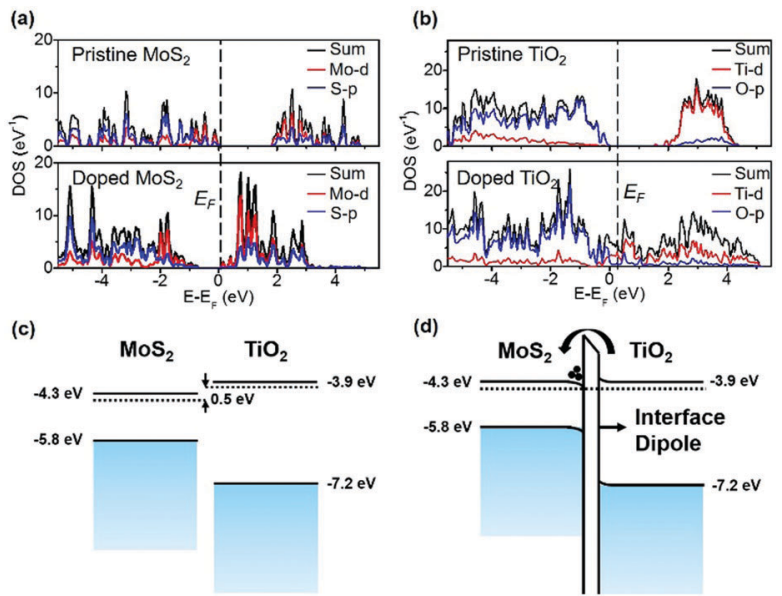

Fig. 4 DFT simulation results of the $n$-doping process and band structures of $\mathrm{MoS}_{2}$ and $\mathrm{TiO}_{2}$. (a) DOS of $1 \mathrm{~L} 1 \mathrm{H}-\mathrm{MoS}_{2}$ before (upper panel) and after doping (lower panel). (b) DOS of anatase $\mathrm{TiO}_{2}$ before (upper panel) and after doping $\mathrm{MoS}_{2}$ (lower panel). (c) Band alignment of $1 \mathrm{~L} \mathrm{MoS}_{2}$ and $\mathrm{TiO}_{2}$ before doping. (d) Band alignment of $1 \mathrm{~L} \mathrm{MoS}_{2}$ and $\mathrm{TiO}_{2}$ after doping. The dashed lines denote the CNL. 
the n-doping effect. Also, the bandgap of $\mathrm{MoS}_{2}$ is obviously shrunken, which is due to the enhanced dielectric screening introduced by $\mathrm{TiO}_{2}$ deposition. From Fig. 4(b), we observe a significant change in the DOS of $\mathrm{TiO}_{2}$ after the n-doping. Compared with the pristine state, the presence of the Ti-5d level in the conduction band is dramatically decreased. This change indicates that electrons are mainly transferred from the Ti-5d level of $\mathrm{TiO}_{2}$ to $\mathrm{MoS}_{2}$, leaving the Ti-5d level anti-bonding. Similar findings regarding the simulation of the $\mathrm{TiO}_{2}$ doping process can be found in the work of N. Kaushik et al. ${ }^{40}$

Fig. 4(c) shows the schematic band structures of $1 \mathrm{~L} \mathrm{MoS}_{2}$ versus anatase $\mathrm{TiO}_{2}$. The CBM and CNL of $\mathrm{MoS}_{2}$ are about $0.5 \mathrm{eV}$ lower than those of $\mathrm{TiO}_{2}$. Driven by this energy difference, electrons in the CB of $\mathrm{TiO}_{2}$ flows into the CB of $\mathrm{MoS}_{2}$, until an interface dipole pointing from $\mathrm{MoS}_{2}$ to $\mathrm{TiO}_{2}$ is built up (Fig. 4(d)) and then the doping process reaches its balance state. As widely acknowledged, electrons in ultrathin $\mathrm{MoS}_{2}$ flakes have originated from surface sulfur vacancies. It is reasonable to expect that this surface effect has a smaller influence on the electronic structure of thicker $\mathrm{MoS}_{2}$ samples. The CNL of $\mathrm{MoS}_{2}$ thin flakes lowers when the thickness of $\mathrm{MoS}_{2}$ increases. Considering the fact that the bandgap of $\mathrm{MoS}_{2}$ decreases with the increase of body thickness, the CNL of thicker $\mathrm{MoS}_{2}$ samples quickly lowers down. For thicker $\mathrm{MoS}_{2}$ samples, the energy difference between its CNL and that of $\mathrm{TiO}_{2}$ enlarges, thus the n-doping effect is more obvious.

\section{Layer-dependent p-doping of $\mathrm{MoS}_{2}$ by $\mathrm{MoO}_{3}$}

$\mathrm{MoO}_{3}$ is one type of TMOs with a relatively low CNL. We choose it for p-doping $\mathrm{MoS}_{2}$. Fig. 5 shows the PL spectra of $1 \mathrm{~L}$ and $3 \mathrm{~L}$ $\mathrm{MoS}_{2}$ before and after $6 \mathrm{~nm}$ thick $\mathrm{MoO}_{3}$ doping. In both cases, a significant decrease of the $\mathrm{X}^{-}$peak spectral weight is observed. The corresponding Raman spectra of these samples are shown in Fig. S3 (ESI $\dagger$ ). No obvious shift in the positions of Raman peaks is observed, suggesting that the interlayer coupling between the $\mathrm{MoO}_{3}$ layer and the $\mathrm{MoS}_{2}$ surface is also very small. Also, it is noteworthy that the dielectric constant of $\mathrm{MoO}_{3}$ is small $(\sim 5.5)$. Therefore, it has a negligible influence on the PL spectra of $\mathrm{MoS}_{2}$.

Electrical characterization of $\mathrm{MoS}_{2}$ FETs is used to further quantify the p-doping effect. Fig. 6(a) is the transfer curve of a $5 \mathrm{~L} \mathrm{MoS} 2$ FET before and after p-doping. After the p-doping, the $I_{\mathrm{ON}}$ at $V_{\mathrm{bg}}=50 \mathrm{~V}$ is decreased 5 times and the threshold voltage $\left(V_{\mathrm{th}}\right)$ is increased by $\sim 24 \mathrm{~V}$. The extracted $n_{2 \mathrm{D}}$ values are $2.3 \times 10^{12} \mathrm{~cm}^{-2}$ before the doping and $1.7 \times 10^{12} \mathrm{~cm}^{-2}$ after the p-doping. The electron density in $\mathrm{MoS}_{2}$ is depleted by about $0.6 \times 10^{12} \mathrm{~cm}^{-2}$,
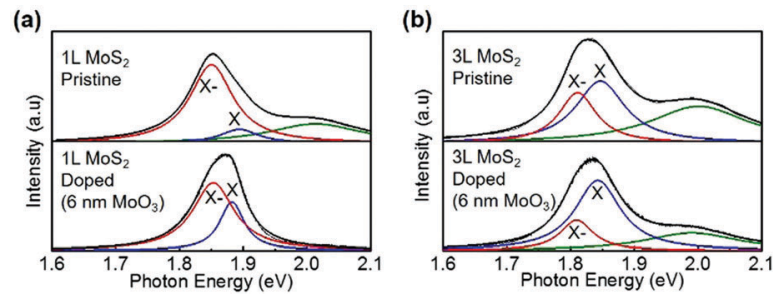

Fig. 5 PL spectra of ultrathin $\mathrm{MoS}_{2}$ flakes before and after being doped by $6 \mathrm{~nm}$ thick $\mathrm{MoO}_{3}$. The thicknesses of $\mathrm{MoS}_{2}$ samples are (a) $1 \mathrm{~L}$ and (b) $3 \mathrm{~L}$.
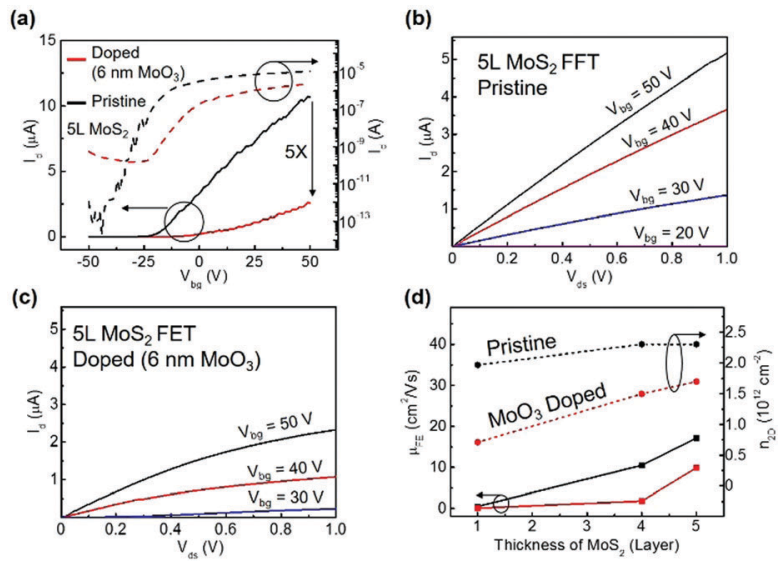

Fig. 6 Electrical characterization of a $\mathrm{MoS}_{2}$ FET for $\mathrm{p}$-doping by $\mathrm{MoO}_{3}$. (a) Transfer curve of a $5 \mathrm{~L} \mathrm{MoS} 2$ FET before (black line) and after (red line) $6 \mathrm{~nm}$ thick $\mathrm{MoO}_{3}$ doping. (b) Output curves of the device before doping. (c) Output curves of the device after $6 \mathrm{~nm}$ thick $\mathrm{MoO}_{3}$ doping. (d) Extracted $\mu_{\mathrm{FE}}$ (left axis) and $n_{2 \mathrm{D}}$ (right axis) of three $\mathrm{MoS}_{2}$ devices before (black line) and after (red line) $6 \mathrm{~nm}$ thick $\mathrm{MoO}_{3}$ doping.

which is comparable to the doping level of $6 \mathrm{~nm}$ thick $\mathrm{TiO}_{2}$ $\left(0.78 \times 10^{12} \mathrm{~cm}^{-2}\right)$. The corresponding output curves of the device are illustrated in Fig. 6(b) (before doping) and Fig. 6(c) (after doping). As expected, we observe a decrease of $I_{\mathrm{ON}}$ in the positive gate range $(0-50 \mathrm{~V})$. The gate voltage required to turn on the channel $\left(V_{\mathrm{ON}}\right)$ is clearly increased from $20 \mathrm{~V}$ to $30 \mathrm{~V}$. The $n_{2 \mathrm{D}}$ and $\mu_{\mathrm{FE}}$ values of $\mathrm{MoS}_{2}$ devices before and after the p-doping are summarized in Fig. 6(d). For thicker $\mathrm{MoS}_{2}$ samples, the depleted electron density is smaller.

This layer dependence can also be ascribed to the decrease of the CNL of $\mathrm{MoS}_{2}$ with the increase of body thickness, as discussed in the case of n-doping by $\mathrm{TiO}_{2}$. Due to the reduced carrier density, electrons undergo a stronger scattering effect from the interface defects, which will significantly reduce the carrier mobility.

DFT calculation results of this p-doping process are shown in Fig. 7. In Fig. 7(a), the Fermi level of $1 \mathrm{~L} \mathrm{MoS}_{2}$ is moved down towards the VB, demonstrating the p-doping effect. The DOS of $\mathrm{MoO}_{3}$ in Fig. 7(b) shows that the population of the Mo-4d orbital is increased after the doping, indicating that electrons are transferred from the Mo-4d orbital in $\mathrm{MoS}_{2}$ to the Mo-4d orbital in $\mathrm{MoO}_{3}$. Fig. 7(c) illustrates the schematic band structure of $1 \mathrm{~L} \mathrm{MoS}_{2}$ versus $\mathrm{MoO}_{3}$. The CNL of $\mathrm{MoS}_{2}$ is $2.2 \mathrm{eV}$ higher than that of $\mathrm{MoO}_{3}$. Due to this energy difference, electrons in the CB of $\mathrm{MoS}_{2}$ are extracted to the $\mathrm{CB}$ of $\mathrm{MoO}_{3}$ until an interface dipole is built up (Fig. 7(d)). When the CNL of $\mathrm{MoS}_{2}$ is lowered down by increasing the body thickness, the energy difference between it and the CNL of $\mathrm{MoO}_{3}$ is decreased, thus leading to a decreased p-doping effect.

\section{Experimental}

\section{Fabrication of $\mathrm{MoS}_{2}$ FETs}

$\mathrm{MoS}_{2}$ crystals were purchased from a commercial producer (2D semiconductors). Mechanically exfoliated $\mathrm{MoS}_{2}$ thin flakes 

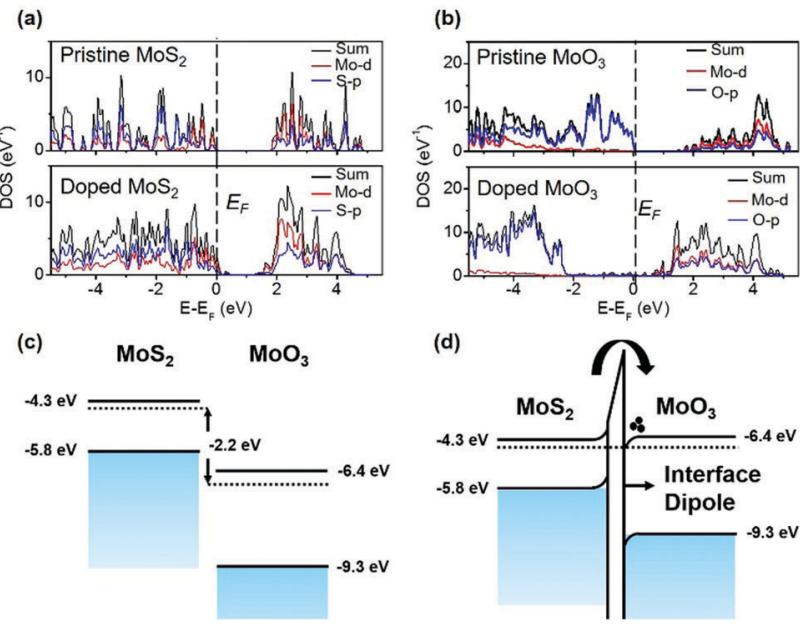

Fig. 7 DFT simulation results of the $\mathrm{p}$-doping process and band structures of $\mathrm{MoS}_{2}$ and $\mathrm{MoO}_{3}$. (a) DOS of $1 \mathrm{~L} 1 \mathrm{H}-\mathrm{MoS}_{2}$ before (upper panel) and after doping (lower panel). (b) DOS of pristine $\mathrm{MoO}_{3}$ before (upper panel) and after doping $\mathrm{MoS}_{2}$ (lower panel). (c) Band alignment of $1 \mathrm{~L} \mathrm{MoS}_{2}$ and $\mathrm{MoO}_{3}$ before doping. (d) Band alignment of $1 \mathrm{~L} \mathrm{MoS}_{2}$ and $\mathrm{MoO}_{3}$ after doping. The dashed lines denote the CNL.

were used to fabricate FETs. Target substrates (300 nm thick $\mathrm{SiO}_{2} / \mathrm{Si}$ wafer) were contacted with Scotch tape and then bathed in Acetone or Isopropyl Alcohol (IPA) at $60{ }^{\circ} \mathrm{C}$ to remove organic residues. Ultrathin flakes $(<10 \mathrm{~nm})$ of $\mathrm{MoS}_{2}$ were identified using an optical microscope. The conventional photo-lithography process was performed, followed by metal deposition and lift-off processes. Metal electrodes (i.e., Ti/Au) were deposited using an electron-beam evaporator at a rate of $\sim 0.7 \AA \mathrm{s}^{-1}$. Electrical characteristics of the FETs were measured using a Keithley 4200-SCS Semiconductor Parameter Analyser.

\section{Deposition of $\mathrm{TiO}_{2}$ and $\mathrm{MoO}_{3}$ oxide layers}

$\mathrm{TiO}_{2}$ oxide layers were deposited on $\mathrm{MoS}_{2}$ using ALD at $150{ }^{\circ} \mathrm{C}$. The sources were tetrakis(dimethylamino)titanium (TDMAT) and $\mathrm{H}_{2} \mathrm{O}$. The deposition rate was $\sim 0.47 \AA$ cycle $^{-1}$. A thermal evaporator was used to deposit $\mathrm{MoO}_{3}$ oxide layers. The source was $\mathrm{MoO}_{3}$ powder. The deposition rate was around $1 \AA \mathrm{s}^{-1}$. The oxide layers are continuous and have small surface roughness.

\section{DFT simulations of the doping process}

DFT calculations were conducted using Quantum ESPRESSO to simulate the charge transfer process between oxide and TMDs. The ion cores were described using projector-augmented wave sets and scalar-relativistic pseudopotentials. The generalized gradient approximation Perdew-Burke-Ernzerhof (PBE) exchangecorrelation was selected as the pseudopotential functional type. $2 \times 1 \times 1$ monolayer $\mathrm{MoO}_{3}$ was constituted on the top of $2 \times 1 \times 11 \mathrm{H}-\mathrm{MoS}_{2}$ along the (001) direction, and $2 \times 1 \times 1$ $\mathrm{TiO}_{2}$ was constituted on the $2 \times 1 \times 11 \mathrm{H}-\mathrm{MoS}_{2}$ along the (001) direction. Each supercell contains a $40 \AA$ vacuum region in the $z$-axis to prevent the effects from the neighbouring slabs. The convergence threshold values on the total energy and force were $10^{-6}$ and $10^{-3}$, respectively. The kinetic energy cut-off for wave functions was $50 \mathrm{Ry}$, and the kinetic energy cut-off for charge density and potential was 200 Ry.

\section{Conclusions}

In summary, we investigate the layer dependence of $\mathrm{n}$ - and p-doping of $\mathrm{MoS}_{2}$ by TMO. $\mathrm{TiO}_{2}$ and $\mathrm{MoO}_{3}$ are used for the n- and p-doping of $\mathrm{MoS}_{2}$, respectively. PL and electrical characterization show that thicker $\mathrm{MoS}_{2}$ samples are more easily n-doped by $\mathrm{TiO}_{2}$; while thinner $\mathrm{MoS}_{2}$ samples are more easily p-doped by $\mathrm{MoO}_{3}$. Furthermore, the doping levels of $6 \mathrm{~nm}$ thick $\mathrm{TiO}_{2}$ and $\mathrm{MoO}_{3}$ are extracted to be $0.78 \times 10^{12} \mathrm{~cm}^{-2}$ and $0.6 \times 10^{12} \mathrm{~cm}^{-2}$, respectively. Our experiments characterized the doping effects and doping levels of the two mostly used oxide dopants $\left(\mathrm{TiO}_{2}\right.$ and $\left.\mathrm{MoO}_{3}\right)$ and shed light on optimizing the doping strategy of TMDs by oxide layers.

\section{Acknowledgements}

This work was supported by the Research Grant Council of Hong Kong (Grant no. PolyU 152145/15E) and the Hong Kong Polytechnic University (Grant no. G-YBPS, 1-ZE25 and 1-ZVDH).

\section{References}

1 B. Radisavljevic, A. Radenovic, J. Brivio, I. V. Giacometti and A. Kis, Nat. Nanotechnol., 2011, 6, 147-150.

2 C. Zhou, Y. Zhao, S. Raju, Y. Wang, Z. Lin, M. Chan and Y. Chai, Adv. Funct. Mater., 2016, 26, 4223.

3 Y. Zhao, J. Qiao, P. Yu, Z. Hu, Z. Lin, S. P. Lau, Z. Liu, W. Ji and Y. Chai, Adv. Mater., 2016, 28, 2399.

4 Z. Lin, Y. Zhao, C. Zhou, R. Zhong, X. Wang, Y. H. Tsang and Y. Chai, Sci. Rep., 2015, 5, 18596.

5 C. Zhou, X. Wang, S. Raju, Z. Lin, D. Villaroman, B. Huang, H. L.-W. Chan, M. Chan and Y. Chai, Nanoscale, 2015, 7, 8695-8700.

6 W. Liu, J. Kang, D. Sarkar, Y. Khatami, D. Jena and K. Banerjee, Nano Lett., 2013, 13, 1983-1990.

7 J.-K. Huang, J. Pu, C.-L. Hsu, M.-H. Chiu, Z.-Y. Juang, Y.-H. Chang, W.-H. Chang, Y. Iwasa, T. Takenobu and L.-J. Li, ACS Nano, 2013, 8, 923-930.

8 Y. Zhang, J. Ye, Y. Matsuhashi and Y. Iwasa, Nano Lett., 2012, 12, 1136-1140.

9 S. Mouri, Y. Miyauchi and K. Matsuda, Nano Lett., 2013, 13, 5944-5948.

10 Y. Zhao, K. Xu, F. Pan, C. Zhou, F. Zhou and Y. Chai, Adv. Funct. Mater., 2016, DOI: 10.1002/adfm.201603484.

11 J. Suh, T.-E. Park, D.-Y. Lin, D. Fu, J. Park, H. J. Jung, Y. Chen, C. Ko, C. Jang and Y. Sun, Nano Lett., 2014, 14, 6976-6982.

12 A. Nipane, D. Karmakar, N. Kaushik, S. Karande and S. Lodha, ACS Nano, 2016, 10, 2128-2137.

13 M. Chen, H. Nam, S. Wi, L. Ji, X. Ren, L. Bian, S. Lu and X. Liang, Appl. Phys. Lett., 2013, 103, 142110.

14 R. Dingle, H. Störmer, A. Gossard and W. Wiegmann, Appl. Phys. Lett., 1978, 33, 665-667. 
15 R. People, J. Bean, D. Lang, A. Sergent, H. Störmer, K. Wecht, R. Lynch and K. Baldwin, Appl. Phys. Lett., 1984, 45, 1231-1233.

16 T. Mimura, S. Hiyamizu, T. Fujii and K. Nanbu, Jpn. J. Appl. Phys., 1980, 19, L225.

17 P. Zhao, D. Kiriya, A. Azcatl, C. Zhang, M. Tosun, Y.-S. Liu, M. Hettick, J. S. Kang, S. McDonnell, S. KC, J. Guo, K. Cho, R. M. Wallace and A. Javey, ACS Nano, 2014, 8, 10808-10814.

18 D. Kiriya, M. Tosun, P. Zhao, J. S. Kang and A. Javey, J. Am. Chem. Soc., 2014, 136, 7853-7856.

19 C.-H. Chen, C.-L. Wu, J. Pu, M.-H. Chiu, P. Kumar, T. Takenobu and L.-J. Li, 2D Mater., 2014, 1, 034001.

20 N. Kaushik, D. Karmakar, A. Nipane, S. Karande and S. Lodha, ACS Appl. Mater. Interfaces, 2015, 8, 256-263.

21 K. F. Mak, K. He, C. Lee, G. H. Lee, J. Hone, T. F. Heinz and J. Shan, Nat. Mater., 2013, 12, 207-211.

22 A. Singh, G. Moody, K. Tran, M. E. Scott, V. Overbeck and G. Berghäuser, Phys. Rev. B, 2016, 93(4), 041401.

23 G. Berghäuser and E. Malic, Phys. Rev. B: Condens. Matter Mater. Phys., 2014, 89, 125309.

24 D. Liu, Y. Guo, L. Fang and J. Robertson, Appl. Phys. Lett., 2013, 103, 183113.

25 S. Kim, M. S. Choi, D. Qu, C. H. Ra, X. Liu, M. Kim, Y. J. Song and W. J. Yoo, 2D Mater., 2016, 3, 035002.

26 E. Rotunno, F. Fabbri, E. Cinquanta, D. Kaplan, M. Longo, L. Lazzarini, A. Molle, V. Swaminathan and G. Salviati, $2 D$ Mater., 2016, 3, 025024.

27 A. Splendiani, L. Sun, Y. Zhang, T. Li, J. Kim, C.-Y. Chim, G. Galli and F. Wang, Nano Lett., 2010, 10, 1271-1275.
28 X. Chen, Z. Wu, S. Xu, L. Wang, R. Huang, Y. Han, W. Ye, W. Xiong, T. Han and G. Long, 2014, arXiv:1407.5365.

29 T. Cheiwchanchamnangij and W. R. Lambrecht, Phys. Rev. B: Condens. Matter Mater. Phys., 2012, 85, 205302.

30 Y. Lin, X. Ling, L. Yu, S. Huang, A. L. Hsu, Y.-H. Lee, J. Kong, M. S. Dresselhaus and T. S. Palacios, Nano Lett., 2014, 14, 5569-5576.

31 L. Yang, X. Cui, J. Zhang, K. Wang, M. Shen, S. Zeng, S. A. Dayeh, L. Feng and B. Xiang, Sci. Rep., 2014, 4, 5649.

32 S. Das, H.-Y. Chen, A. V. Penumatcha and J. Appenzeller, Nano Lett., 2012, 13, 100-105.

33 H.-Y. Park, M.-H. Lim, J. Jeon, G. Yoo, D.-H. Kang, S. K. Jang, M. H. Jeon, Y. Lee, J. H. Cho and G. Y. Yeom, ACS Nano, 2015, 9, 2368-2376.

34 D. M. Sim, M. Kim, S. Yim, M.-J. Choi, J. Choi, S. Yoo and Y. S. Jung, ACS Nano, 2015, 9, 12115-12123.

35 B. Radisavljevic and A. Kis, Nat. Mater., 2013, 12, 815-820. 36 D.-H. Kang, J. Shim, S. K. Jang, J. Jeon, M. H. Jeon, G. Y. Yeom, W.-S. Jung, Y. H. Jang, S. Lee and J.-H. Park, ACS Nano, 2015, 9, 1099-1107.

37 P. H. Ho, C. H. Chen, F. Y. Shih, Y. R. Chang, S. S. Li, W. H. Wang, M. C. Shih, W. T. Chen, Y. P. Chiu and M. K. Li, Adv. Mater., 2015, 27, 7809-7815.

38 Q. Wang, S. Ge, X. Li, J. Qiu, Y. Ji and J. Feng, ACS Nano, 2013, 7(12), 11087-11093.

39 D. Lagarde, L. Bouet, X. Marie, C. R. Zhu, B. L. Liu, T. Amand, P. H. Tan and B. Urbaszek, Phys. Rev. Lett., 2014, 112(4), 047401.

40 N. Kaushik, D. Karmakar, A. Nipane, S. Karande and S. Lodha, ACS Appl. Mater. Interfaces, 2015, 8(1), 256-263. 